\title{
ON THE STRUCTURE OF BANACH SPACES WITH CERTAIN GEOMETRIC PROPERTIES
}

\author{
D. SOYBAS, Z. ARGÜN AND H. AKAY
}

\begin{abstract}
Let $X$ be a Banach space whose dual has the property $\left(\mathrm{V}^{*}\right)$ and $Y$ be a Banach space whose dual does not contain an isomorphic copy of $l_{\infty}$. We showed that every bounded linear operator from $Y$ to $X^{*}$ is weakly compact. Several results were given on dual Banach spaces concerning some geometric properties.
\end{abstract}

\section{INTRODUCTION}

There has been many geometric properties of Banach spaces defined to investigate the structures of Banach spaces, instead of analysing them one by one. In his fundamental papers $[8,9]$, Pelczynski introduced properties $(u),(V),\left(V^{*}\right)$ as tools to study the structure of Banach spaces. In the latter paper, the so called properties $(\mathrm{V})$ and $\left(\mathrm{V}^{*}\right)$ are defined by the coincidence of $(\mathrm{V})$ or $\left(\mathrm{V}^{*}\right)$ sets with the weakly relatively compact sets. Many important Banach spaces properties are (or can be) defined in the same way; that is, by the coincidence of two classes of bounded set. For any Banach space $X$, corresponding to the decomposition $X^{* * *}=X^{*} \oplus X^{\perp}$, we have a natural projection $p: X^{* * *} \rightarrow X^{*}$ that sends each $\mu \in X^{* * *}$ to its restriction to $X$, where $X$ is regarded as a subspace of its bidual $X^{* *}$. By a well-known result of R. Phillips [3], the canonical projection $p: c_{0}^{* * *} \rightarrow c_{0}^{*}$ is sequentially weak*-tonorm continuous. This fact motivated W. Freedman and A. Ülger to introduce the so called Phillips and weak Phillips property [5]: A Banach space $X$ is said to have the (weak) Phillips property if the natural projection $p: X^{* * *} \rightarrow X^{*}$ is sequentially weak*-to-norm (weak*-to-weak) continuous. The above mentioned authours studied these two properties and their hereditary versions, to a certain extent, in the same paper. Then, further results on the weak Phillips property was given in a paper by A. Ülger [12].

In this paper, we are concerned with the study of the class of $\left(\mathrm{V}^{*}\right)$ Banach spaces and its relationship with other related Banach spaces' geometric properties. The main result in our paper is that every operator from a Banach space $Y$ whose dual does not contain an isomorphic copy of $l_{\infty}$, to a dual Banach space $X^{*}$ with the property $\left(\mathrm{V}^{*}\right)$ is weakly compact (i.e., every operator $T: X \rightarrow X^{*}$ is weakly

Received by the editors April 05, 2005, Accepted: June 23, 2005.

1991 Mathematics Subject Classification. 2000 Mathematics Subject Classification. : Primary 43B20, 46B03; Secondary 46L05

Ke:y words and phrases. (V) and $\left(\mathrm{V}^{*}\right)$ properties of Pelczyuski, Grothendieck property, the weak Phillips property, unconditionally convergence, weak compactness. 
compact), which extends previous result of E. and P. Saab [11].

\section{Notations:}

We shall try to follow the standart notations in Banach space theory, as in [12]. In order to prevent any doubt, we shall fix some terminology. If $X$ is a Banach space, $B(X)$ will be its closed unit ball and $X^{*}$ its topological dual. The word operator will always mean linear bounded operator, and respectively $L(X, Y)$ and $W(X, Y)$ will stand for the Banach spaces of respectively all operators and all weakly compact operators from $X$ to $Y$. A series $\sum x_{n}$ in $X$ is said to be weakly unconditionally Cauchy (w.u.c. in short) if $\sum\left|x^{\prime}\left(x_{n}\right)\right|<\infty$ for every $x^{\prime} \in X^{*}$. An operator $T$ is said to be unconditionally converging if sends w.u.c. series $\sum x_{n}$ in $X$ into unconditionally converging series $\sum T\left(x_{n}\right) . U(X, Y)$ denotes the set of all unconditionally converging operators from $X$ to $Y$. Every weakly compact operator is unconditionally converging; that is, $W(X, Y) \subset U(X, Y)[9]$.

\section{2. $\left(\mathrm{V}^{*}\right.$ ) Sets and $\left(\mathrm{V}^{*}\right)$ Spaces in Relation with Some Other Properties}

Definition 1.1:A subset $K$ of a Banach space $X$ is called a $\left(\mathrm{V}^{*}\right)$ set if for every w.u.c. series $\sum x_{n}^{\prime}$ in $X^{*}$, the equation $\lim _{n} \sup \left\{\left|\left\langle x_{n}^{\prime}, x\right\rangle\right|: x \in K\right\}=0$ is satisfied. It is obvious that a $\left(\mathrm{V}^{*}\right)$ set is bounded. Also, every relatively weakly compact set is a $\left(\mathrm{V}^{*}\right)$ set. The definition of a $\left(\mathrm{V}^{*}\right)$ space was introduced by Pelczynski in [9] as: A Banach space $X$ is said to have the property $\left(\mathrm{V}^{*}\right)$ of Pelczynski if every $\left(\mathrm{V}^{*}\right)$ set in $X$ is relatively weakly compact. The space $l_{1}$ and abstract $L$-spaces have the property $\left(\mathrm{V}^{*}\right)[9]$.

Definition 1.2: A subset $K$ of a Banach space $X^{*}$ is called a (V) set if for every w.u.c. $\sum x_{n}$ in $X$, the equation $\lim _{n} \sup \left\{\left|<x_{n}, x^{\prime}\right\rangle \mid: x^{\prime} \in K\right\}=0$ is satisfied.

It is obvious that a (V) set is bounded. Also, every relatively weakly compact set is a (V) set. The definition of a (V) space was introduced by Pelczynski in [9] as: A Banach space $X$ is said to have the property (V) of Pelczynski if every (V) set in $X^{*}$ is relatively weakly compact. The space $c_{0}$ and $C(K)$ spaces have the property (V) [9]. We need the following lemma before giving our main result Theorem 1.4.

Lemma 1.3. Let $X$ be a Banach space and $Y$ be a dual Banach space not containing an isomorphic copy of $l_{\infty}$. Then the equality $U\left(X^{*}, Y\right)=L\left(X^{*}, Y\right)$ holds.

Proof: Let $X$ be a Banach space and $Y$ be a dual Banach space not containing an isomorphic copy of $l_{\infty}$. Suppose that we have an operator $T: X^{*} \rightarrow Y$ which is unconditionally converging. Then by a theorem of [1], there exists a subspace $\mathrm{M}$ of $X^{*}$ such that $M$ isomorphic to $c_{0}$ and the restriction $T \mid M$ of $T$ is an isomorphism on $M$. Since $M \cong c_{0}$ we have $M^{* *} \cong l_{\infty}$. Considering the natural injection $i: M \rightarrow X^{*}$ and the natural projection $p: X^{* * *} \rightarrow X^{*}$, take the composition $T$ opoi $^{* *}: M^{* *} \rightarrow Y$. For the sake of shortness, write it as $S=T o p o i^{* *}$. Since $M^{* *} \cong l_{\infty}$ is an injective space [6] and every operator from an injective space to a space not containing an isomorphic copy of $l_{\infty}$ is weakly compact from Corollary 1.4 of Rosenthal [10], $S$ is weakly compact. Then the restriction $T\left|M=\left(T o p o i^{* *}\right)\right| M$ is weakly compact. Since $T\left|M=\left(T o p o i^{* *}\right)\right| M=T \mid M$ holds, $T \mid M$ is weakly compact 
which means $(T \mid M)^{* *}\left(M^{* *}\right) \subset c_{0}$ by Goldstein theorem. However, in this case, we would have the contradiction $l_{\infty} \subset c_{0}$ since the restriction $T \mid M$ is an isomorphism.

Theorem 1.4: Let $X$ be a Banach space whose dual has the property $\left(\mathrm{V}^{*}\right)$ and $Y$ be a Banach space whose dual not containing an isomorphic copy of $l_{\infty}$. Then the equality $W\left(Y, X^{*}\right)=L\left(Y, X^{*}\right)$ holds.

Proof:Under the assumptions of the hypthesis, let an operator $T: Y \rightarrow X^{*}$ be given. Consider the adjoint operator $T^{* *}: X^{* *} \rightarrow Y^{*}$. Since every operator from a dual space to another Banach space not containing an isomorphic copy of $l_{\infty}$ is unconditionally converging according to above lemma, the operator $T^{* *}: X^{* *} \rightarrow Y^{*}$ is unconditionally converging. Let $y_{m}$ be an arbitrary bounded sequence in $X$ and let $\sum x_{n}^{\prime \prime}$ be an arbitrary w.u.c. series in $X^{* *}$. Take $K=T\left(y_{m}\right)$. Since is an unconditionally converging operator, $T^{* *}: X^{* *} \rightarrow Y^{*}$ is unconditionally converging series. Thus, by the condition $(\mathrm{H})$ in [7],

$$
\begin{gathered}
\lim _{n} \sup \left\{\left|<x_{n}^{\prime \prime}, x^{\prime}>\right|: x^{\prime} \in K\right\}=\lim _{n} \sup \left\{\left|<x_{n}^{\prime \prime}, T\left(y_{m}\right)>\right|: m \in I N\right\} \\
=\lim _{n} \sup \left\{\left|<T^{*}\left(x_{n}^{\prime \prime}\right), y_{m}>\right|: m \in I N\right\}=0
\end{gathered}
$$

Hence the set $K$ is a $\left(\mathrm{V}^{*}\right)$ set in $X^{*}$. Since the dual $X^{*}$ has the property $\left(\mathrm{V}^{*}\right)$, the set $K$ is relatively weakly compact, which means the sequence $T\left(y_{m}\right)$ has a weakly convergent (to an element of $X^{*}$ ) subsequence. Hence the operator $T: Y \rightarrow X^{*}$ is weakly compact.

Theorem 1.4 extends previous result of $E$. and P. Saab [11], which we give as a corollary as follows:

Corollary 1.5: Let $X$ be a Banach space whose dual has the property $\left(\mathrm{V}^{*}\right)$. Since $X^{*}$ has the property $\left(\mathrm{V}^{*}\right)$ and every $\left(\mathrm{V}^{*}\right)$ space is weakly sequentially complete (w.s.c.), $X^{*}$ does not contain an isomorphic copy of $l_{\infty}$ (it is well-known that the space $l_{\infty}$ is not w.s.c. and every closed subspace of a w s.c. space is also w.s.c.). Then every operator $T: X \rightarrow X^{*}$ is weakly compact

Since when a Banach space $X$ has the property (V) then the dual $X^{*}$ has the property $\left(V^{*}\right)$, we immediately have the following result.

Corollary 1.6: Let $X$ be a Banach space with the property (V). Then every operator $T: X \rightarrow X^{*}$ is weakly compact [11].

E. and P. Saab, in [11], introduced a property so-called (W) property. A Banach space $X$ has the property (W) if every operator $T: X \rightarrow X^{*}$ is weakly compact. By the above corollary, every Banach space whose dual has the property $\left(V^{*}\right)$ has (W) property too.

\section{3. (V2), Weak Phillps and Grothendieck Properties for Dual Banach SPACES}

In literature, an equal definition of $(\mathrm{V})$ property for Banach spaces are sometimes given as: A Banach space $X$ is said to have (V) property if every unconditionally 
converging operator on $X$ is weakly compact.

Definition 2.1: A Banach space $X$ is said to have $\left(V_{1}\right)$ property if every unconditionally converging operator $T: X \rightarrow c_{0}$ is weakly compact. It is clear that every (V) space is $\left(V_{1}\right)$ space. Similarly; A Banach space $X$ is said to have $\left(V_{2}\right)$ property if , for every separable Banach space $Y$, every unconditionally converging operator $T: X \rightarrow Y$ is weakly compact. From the definitions, it is obvious that every $\left(V_{2}\right)$ space is $\left(V_{1}\right)$ space.

Definition 2.2: A Banach space $X$ is said to have the (weak) Phillips property if the natural projection $p: X^{* * *} \rightarrow X^{*}$ is sequentially weak*-to-norm (weak*-toweak) continuous.

Below Theorem 2.3 due to W. Freedman and A. Ülger [5] was the first to make characterisation of Banach spaces having the Phillips and the weak Phillips property.

Theorem 2.3: A Banach space $X$ has the (weak) Phillips property if and only if , for every operator $T: X^{* *} \rightarrow c_{0}$, the restriction $\tilde{T}: X \rightarrow c_{0}$ is (weak) compact.

Theorem 2.4: Let $X$ be a Banach space. If either $X$ or $X^{* *}$ has the $\left(V_{2}\right)$ property then the space $X$ has the weak Phillips property.

Proof: Let $X$ have $\left(V_{2}\right)$ property and an operator $T: X^{* *} \rightarrow c_{0}$ be given. Since the space $c_{0}$ does not contain a copy of $l_{\infty}$, by Lemma 1.3 the operator $T: X^{* *} \rightarrow c_{0}$ is unconditionally converging and hence, its restriction to $X, \tilde{T}$ is unconditionally converging. Since the space $c_{0}$ is separable and $X$ has the property $\left(V_{2}\right)$, the restriction $\tilde{T}$ is weakly compact. Hence $X$ has the weak Phillips property by Theorem 2.3. By the same argument, if the bidual $X^{* *}$ has the property $\left(V_{2}\right)$ the operator $T: X^{* *} \rightarrow c_{0}$, and hence, $\tilde{T}$ is unconditionally converging.

We show, by the following example, that Banach spaces having the weak Phillips property does not need to have the property $\left(V_{2}\right)$.

Example 2.5: Consider the space $Y$ constructed by Bourgain and Delbean in [2]. Since $Y^{*} \cong l_{1}$, the space $Y$ has the weak Phillips property. However, since the space $Y$ is not reflexive and does not contain a copy of $c_{0}$ it fails to the property (V) and hence fails to have the property $\left(V_{2}\right)$.

Recall that a Banach space $X$ is said to have the Grothendieck property if, for every separable Banach space $Y$, every operator $T: X \rightarrow Y$ is weakly compact [4]. Now we can give the following proposition.

Proposition 2.6: If a dual space $X$ has the property $\left(V_{2}\right)$ then it has the Grothendieck property.

Proof: Let $Y$ be a Banach space such that $X=Y^{*}$ and $Z$ be a separable Banach space. Take an operator $T: Y^{*} \rightarrow Z$. Since $Z$ is separable it does not contain a 
copy of $l_{\infty}$, the operator $T: Y^{*} \rightarrow Z$ is unconditionally converging by Lemma 1.3. Now since $X$ has the property $\left(V_{2}\right)$ the operator $T: Y^{*} \rightarrow Z$ is weakly compact. Hence by the definition $X$ has the Grothendieck property.

Corollary 2.7: Since A Banach space with the property (V) has the property $\left(V_{2}\right)$, Banach spaces with the property $(\mathrm{V})$ have the Grothendieck property.

Note that Proposition 2.5 is valid even under the hypothesis the space $X$ has the property $\left(V_{1}\right)$. For, in this case, an operator $T: Y^{*} \rightarrow c_{0}$ is weakly compact also.

W. Freedman and A. Ülger [5] proved below Theorem 2.9 by using Theorem 2.3 that characterizes the class of Banach space having the Phillips and the weak Phillips property. Whereas, we give directly an elementary proof which only use Definition 2.2 of the weak Phillips property. Firstly, we give following Theorem 2.8, so-called Sobczyk Theorem in literature.

Theorem 2.8: Let $X$ be a separable Banach space. If $X$ contains a closed linear subspace $Y$ isomorphic to $c_{0}$ then there exists a bounded linear projection $q: X \rightarrow Y[3]$.

Theorem 2.9: If a Banach space $X$ has the Pelczynski's property (V), then $X$ has the weak Phillips property.

Proof: Let the space $X$ have the property $(\mathrm{V})$. By the definition, it is enough to show that the natural projection $p: X^{* * *} \rightarrow X^{*}$ is sequentially weak*-to-weak continuous. Let $\left(x_{n}^{\prime \prime \prime}\right)$ be a weak ${ }^{*}-0$ sequence in the third dual $X^{* * *}$. Then for every $x^{\prime \prime} \in X^{* *}$, we have $\left\langle x^{\prime \prime}, x_{n}^{\prime \prime \prime}>\longrightarrow 0\right.$. Hence, the operator defined by $\left.T\left(x^{\prime \prime}\right)=\left(<x^{\prime \prime}, x_{n}^{\prime \prime \prime}\right\rangle\right)$, for each $x^{\prime \prime} \in X^{* *}$, is obviously bounded and linear. Let the sequence $\left(e_{n}^{*}\right)$ be a standart unit vector basis of $l_{1}$. Recall that "weak compactness of an operator $T: X \rightarrow c_{0}$ is equal to being weak-0 for the sequence $T^{*}\left(e_{n}^{*}\right)^{\text {" }}$ [3]. Now for each $x^{\prime \prime} \in X^{* *}$;

$$
<x^{\prime \prime}, T^{*}\left(e_{n}^{*}\right)>=<T\left(x^{\prime \prime}\right), e_{n}^{*}>=<\left(<x^{\prime \prime}, x_{n}^{\prime \prime \prime}>\right), e_{n}^{*}>=<x^{\prime \prime}, x_{n}^{\prime \prime \prime}>
$$

holds. By these equalities we have $x_{n}^{\prime \prime \prime}=T^{*}\left(e_{n}^{*}\right)$. By means of taking into account the equality $\tilde{T}^{*}=p T^{*}$ and applying the projection $p$, we have $p\left(x_{n}^{\prime \prime \prime}\right)=\tilde{T}^{*}\left(e_{n}^{*}\right)$. Assume that $p\left(x_{n}^{\prime \prime \prime}\right)$ is not a weak-0 sequence. Then from the above recalling, the operator $\tilde{T}$ is not weakly compact. Since the space $X$ has the property (V), $X$ has a subspace $K$ isomorhic to $c_{0}$ such that $\tilde{T} \mid K$, the restriction of $\tilde{T}$ to $K$, is an isomorphism [1]. Since $c_{0}$ is separable by Sobczyk Theorem there exists a projection $q: c_{0} \rightarrow \tilde{T}(K)$. Since the restriction $\tilde{T} \mid K$ is isomorphism, the inverse $\tilde{T} \mid K^{-1}$, exists. If we write $R=\tilde{T} \mid K^{-1}$ oqoT then $R: X^{* *} \rightarrow K$ is a projection. Since a Banach space that is complemented in a dual space is complemented in its bidual, by considering $K \cong c_{0}$, we conclude $c_{0}$ is complemented in $l_{\infty}$. Yet, this is a contradiction. Then our assumption $p\left(x_{n}^{\prime \prime \prime}\right)$ is not a weak-0 sequence is false. Hence the natural projection $p: X^{* * *} \rightarrow X^{*}$ is sequentially weak*-to-weak continuous, that is, $X$ has the weak Phillips property. 
OZET: $X$ duali $\left(\mathrm{V}^{*}\right)$ ठzelligine sahip bir Banach uzayı ve $Y$ de duali $l_{\infty}$ uzayının izomorfik bir kopyasın ihtiva etmeyen bir Banach uzayı olsun. Bu şartlar altında $Y$ uzayından $X^{*}$ dualine tanımlanan her sınırlı lineer operatörün zayıf kompakt olduğunu gösterdik. Ayrıca dual Banach uzayları üzerinde bazı geometrik ozzelliklerle ilgili birkaç sonuç verilmistir.

\section{REFERENCES}

[1] Bessaga, C. and Pelczynski, A. On bases and unconditionally convergence of series in Banach spaces, Studia Math. 17 (1958), 151-160.

[2] Bourgain, J., and Delbaen, F., A class of special spaces, Acta Math. 145 (1981), 155-176.

[3] Dirstel, J., Sequences and series in Banach spaces, 1993, Newyork: Springer-Verlag.

[4] Diestel, J. and Uhl, J.J., Vector Measures, Math. Surveys 15, 1977, Amer. Math. Soc., Providence.

[5] Freedman, W., and Ülger A., The Phillips Properties, Amer. Math. Soc. Proc. 128 (1997), 2137-2145.

[6] Lindenstrauss, J. and Tzafriri, L., Classical Banach Spaces, 1973, Lecture Notes in Mathematics 338: Springer- Verlag.

[7] McArthur, Ch. W., A note on subseries convergence, Bull. Amer. Math. Soc. 67 (1961), 540-545.

[8] Pelc:aynski, A., A connection between weakly unconditionally convergence and weakly completeness of Banach spaces, Bull. Acad. Polon. Sci. Ser. Ser. Sci. Math. Astronom. phys. 6 (1958), 251-253.

[9] Pelczynski, A., Banach spaces on which every unconditionally converging operator is weakly compact, Bull. Acad. Polon. Sci. Ser. Ser. Sci. Math. Astronom. phys. 10 (1962), 641-648.

[10] Rosenthal, H., On relatively disjoint families of measures with some applications to Banach space theory, Studia Math. 37 (1970), 13-36.

[11] Saab, E. and Saab,V.,Extensions of some classes of operators and applications, Rocky Mountain J. Math. 23 (1) (1993), 319-337.

[12] Ülger, A., The weak Phillips properties, Colloquium Math. 87 (2001), 147-158.

Current address: D. SOYBAŞ, Z. ARGÜN, H. AKAY. Department of Mathematics, Faculty of Education, Gazi University, Teknikokullar, 06500, Ankara, Turkey,

E-mail address: danyal@gazi.edu.tr,ziya@gazi.edu.tr, hayri@gazi.edu.tr, 